\title{
BASIC SCIENCE ARTICLE \\ Blood metabolomics in infants enrolled in a dose escalation pilot trial of budesonide in surfactant
}

\author{
Philip L. Ballard ${ }^{1}$, Dara Torgerson ${ }^{1}$, Rajan Wadhawan ${ }^{2}$, Mark L. Hudak ${ }^{3}$, Joern-Hendrik Weitkamp ${ }^{4}$, Julia Harris ${ }^{5}$, Jeanette Asselin ${ }^{6}$, \\ Cheryl Chapin ${ }^{1}$, Roberta A. Ballard ${ }^{1}$ and Cindy T. McEvoy ${ }^{5}$
}

BACKGROUND: The pathogenesis of BPD includes inflammation and oxidative stress in the immature lung. Corticosteroids improve respiratory status and outcome, but the optimal treatment regimen for benefit with low systemic effects is uncertain.

METHODS: In a pilot dose escalation trial, we administered $\leq 5$ daily doses of budesonide in surfactant to 24 intubated premature infants (Steroid And Surfactant in ELGANs (SASSIE)). Untargeted metabolomics was performed on dried blood spots using UPLC-MS/MS. Tracheal aspirate IL-8 concentration was determined as a measure of lung inflammation.

RESULTS: Metabolomics data for 829 biochemicals were obtained on 121 blood samples over $96 \mathrm{~h}$ from 23 infants receiving 0.025 , 0.05 , or $0.1 \mathrm{mg}$ budesonide/ $\mathrm{kg}$. Ninety metabolites were increased or decreased in a time- and dose-dependent manner at $q \leq 0.1$ with overrepresentation in lipid and amino acid super pathways. Different dose response patterns occurred, with negative regulation associated with highest sensitivity to budesonide. Baseline levels of 22 regulated biochemicals correlated with lung inflammation (IL-8), with highest significance for sphingosine and thiamin.

CONCLUSIONS: Numerous metabolic pathways are regulated in a dose-dependent manner by glucocorticoids, which apparently act via distinct mechanisms that impact dose sensitivity. The findings identify candidate blood biochemicals as biomarkers of lung inflammation and systemic responses to corticosteroids.

Pediatric Research (2021) 90:784-794; https://doi.org/10.1038/s41390-020-01343-z

\section{IMPACT:}

- Treatment of premature infants in respiratory failure with $0.1 \mathrm{mg} / \mathrm{kg}$ intra-tracheal budesonide in surfactant alters levels of $\sim 11 \%$ of detected blood biochemicals in discrete time- and dose-dependent patterns.

- A subset of glucocorticoid-regulated biochemicals is associated with lung inflammatory status as assessed by lung fluid cytokine concentration.

- Lower doses of budesonide in surfactant than currently used may provide adequate anti-inflammatory responses in the lung with fewer systemic effects, improving the benefit:risk ratio.

\section{INTRODUCTION}

Extremely low gestational age newborns (ELGANs) are at high risk for bronchopulmonary dysplasia (BPD), a disorder that is associated with significant complications in the neonatal intensive care unit and with increased respiratory exacerbations, altered pulmonary function, and neurodevelopmental abnormalities after discharge. The pathogenesis for BPD is multifactorial, but important components are inflammation and oxidative stress that result from intrauterine events as well as postnatal hypoxia, hyperoxia, ventilator trauma, and/or infection in susceptible infants with immature lungs. ${ }^{1,2}$

Corticosteroids are powerful anti-inflammatory agents and are one of the few therapies shown to improve respiratory status in premature infants with respiratory failure and reduce BPD. Postnatal corticosteroid treatment regimens have used both systemic and inhaled corticosteroids with varying benefit and adverse outcomes such as intestinal perforation and increased cerebral palsy. ${ }^{3,4}$ Because glucocorticoids have multiple differentiation, metabolic, and anti-inflammatory effects, there is a need to determine the dose- and time-dependency of responses to optimize the benefit:risk ratio. There is also interest in delivering postnatal corticosteroids topically to the lungs with the goal of reducing systemic responses while optimizing anti-inflammatory effects in the lung. Yeh et al..$^{5,6}$ conducted two unblinded pilot randomized trials of budesonide $(0.25 \mathrm{mg} / \mathrm{kg}$ ) mixed in surfactant (Survanta) vs surfactant alone administered soon after delivery to infants $<1500 \mathrm{~g}$ with severe respiratory distress syndrome. Both trials demonstrated a significant decrease in the primary outcome of death or BPD at 36 weeks postmenstrual age without apparent adverse neurodevelopmental effects at 2 years of age. While these surfactant-based trials are encouraging, further investigation is required to optimize efficacy and safety and to establish the

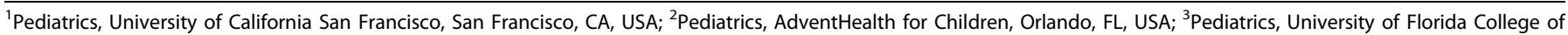

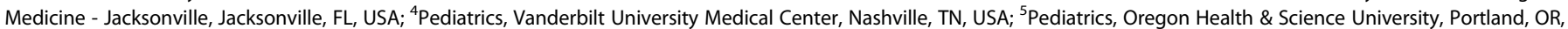
USA and ${ }^{6}$ Oakland Children's Hospital, Oakland, CA, USA

Correspondence: Philip L. Ballard (Phil.ballard@ucsf.edu)

Received: 1 July 2020 Revised: 4 December 2020 Accepted: 9 December 2020

Published online: 19 January 2021 
lowest effective dose. One approach is metabolomics, and currently there is limited information on metabolic responses to glucocorticoids in infants. ${ }^{7}$

Metabolomics analysis, which involves separation of low molecular weight biochemicals by high-performance chromatography and identification/quantification of known compounds by mass spectrometry, has recently emerged as an important component of systems biology for defining the deep phenotype of individuals in pursuit of precision medicine. This approach can identify metabolic derangements and biomarkers, establish new therapeutic biochemical targets, and provide a means to follow responses to treatments. ${ }^{8}$ In the area of lung diseases, metabolomics studies have described candidate biomarkers that await validation. ${ }^{9,10}$

We recently reported a dose escalation pilot study (Steroids and Surfactant in ELGANs (SASSIE)) to determine the optimal dose (and frequency) of instilled budesonide suspended in surfactant to effectively and safely treat intubated infants at high risk for BPD. ${ }^{11}$ We found that lower doses of budesonide $(0.025,0.05$, and 0.1 $\mathrm{mg} / \mathrm{kg}$ ) than previously administered $(0.25 \mathrm{mg} / \mathrm{kg})$ were all similarly effective in decreasing the lung inflammatory response that contributes to BPD in intubated preterm infants. By contrast, there was a dose-response relationship for the total number of budesonide-regulated blood metabolites in an untargeted metabolomics analysis. In the current metabolomics study, we describe positive and negative changes over time in a subset of blood metabolites, differences between biochemicals in apparent sensitivity to corticosteroid, and selected budesonide-regulated metabolites that are associated with the level of lung inflammation.

\section{METHODS}

Study design and participants

SASSIE was a phase $\mathrm{I} / \mathrm{II}$ open label dose escalation trial conducted at 4 US hospitals (Clinicaltrials.gov: NCT 02907593). The primary objective of the study was to determine the clinical and antiinflammatory responses by monitoring infant clinical outcomes up to 28 days of age and measuring blood budesonide and tracheal aspirate (TA) cytokine levels. We treated 8 intubated ELGANs at risk for BPD with budesonide in surfactant (calfactant, $3 \mathrm{ml} / \mathrm{kg}$ ) at each of three dosing levels: $0.025,0.05$, and $0.1 \mathrm{mg} / \mathrm{kg}$ daily for $\leq 5$ days. Inclusion criteria were infants with a gestational age of $23 \%$ to $27 \% / 7$ weeks who were mechanically ventilated between postnatal day of life 3 and 14 with extubation not anticipated within $24 \mathrm{~h}$. Infants who were clinically unstable; had received systemic corticosteroids; had received indomethacin, ibuprofen, or acetominophen to treat a patent ductus arteriosus (PDA); or had congenital malformations or chromosomal anomalies were excluded. Detailed clinical data, including respiratory parameters for calculation of the respiratory severity score $\left(\mathrm{RSS} ; \mathrm{FiO}_{2} \times\right.$ mean airway pressure) were collected. The research protocol was approved by the Institutional Review Boards (IRBs) of the participating institutions, and a parent of each infant provided written informed consent. Further details of trial design, infant demographics, and outcomes have been reported. ${ }^{11}$

\section{Materials and procedures}

TAs were collected from the endotracheal tube using saline instillation just prior to (baseline) and $12 \mathrm{~h}$ after the first study dose, just before each of the subsequent doses, and $48 \mathrm{~h}$ after the fifth dose if the infant remained intubated. Dried blood spot (DBS) samples were collected $15 \mathrm{~min}, 1 \mathrm{~h}$, and $4 \mathrm{~h}$ after the first dose of budesonide and at subsequent 24-h intervals after the first dose, and punches were taken for the measurement of budesonide as described ${ }^{11}$; the DBS were stored at $-20^{\circ} \mathrm{C}$ and subsequently additional punches, as available, were obtained for metabolomics analysis. We did not collect a time zero blood sample because budesonide is not present before treatment and metabolic responses to corticosteroid are delayed; thus we could not justify the additional blood collection for the IRB. All DBS samples for each infant were collected from either a central umbilical line if present or by heel stick for a total of $\leq 7$ samples/infant. There was no effect of blood source on metabolomics results by principal component analysis (data not shown).

Human chemokine C-X-C chemokine motif ligand 8/interleukin (IL)-8 was quantified in TA samples by enzyme-linked immunosorbent assay (R\&D Systems, Minneapolis, MN), and secretory human $\lg \mathrm{A}$ was used for normalization to lung fluid as described. ${ }^{11}$ Changes in IL-8 concentrations in response to budesonide were representative of ten other assayed cytokines/ chemokines in TA, consistent with IL-8 as a marker of lung inflammatory status. ${ }^{11}$

Untargeted (global) metabolomics analysis was performed on 121 available DBS samples from 23 infants using a punch from each DBS. This procedure uses liquid chromatography (LC) with different elution conditions based on compound polarity and hydrophilicity to separate thousands of low-molecular weight ( $<1500 \mathrm{da}$ ) biochemicals of both endogenous (amino acids, peptides, carbohydrates, nucleotides, lipids) and exogenous (dietary, drugs, toxins) origin. Using tandem mass spectrometry, compounds corresponding to known standards are identified and quantitated. ${ }^{8,10}$

Ultrahigh performance LC-tandem mass spectroscopy (UPLCMS/MS) was performed by Metabolon Inc. (Morrisville, NC) as described. ${ }^{12}$ All methods utilized a Waters ACQUITY UPLC and a Thermo Scientific Q-Exactive high-resolution/accurate mass spectrometer interfaced with a heated electrospray ionization (HESI-II) source and Orbitrap mass analyzer operated at 35,000 mass resolution. Each reconstitution solvent contained a series of standards at fixed concentrations to ensure injection and chromatographic consistency. One aliquot was analyzed using acidic positive ion conditions, chromatographically optimized for more hydrophilic compounds. In this method, the extract was gradient eluted from a C18 column (Waters UPLC BEH C18$2.1 \times 100 \mathrm{~mm}, 1.7 \mu \mathrm{m}$ ) using water and methanol, containing $0.05 \%$ perfluoropentanoic acid (PFPA) and $0.1 \%$ formic acid (FA). Another aliquot was also analyzed using acidic positive ion conditions optimized for more hydrophobic compounds. In this method, the extract was gradient eluted from the same C18 column using methanol, acetonitrile, water, 0.05\% PFPA, and $0.01 \% \mathrm{FA}$ and was operated at an overall higher organic content. Another aliquot was analyzed using basic negative ion optimized conditions using a separate C18 column. These extracts were gradient eluted using methanol and water, with $6.5 \mathrm{mM}$ ammonium bicarbonate at $\mathrm{pH}$ 8. The fourth aliquot was analyzed via negative ionization following elution from a HILIC column (Waters UPLC BEH Amide $2.1 \times 150 \mathrm{~mm}, 1.7 \mu \mathrm{m}$ ) using a gradient consisting of water and acetonitrile with $10 \mathrm{mM}$ ammonium formate, $\mathrm{pH}$ 10.8. The MS analysis alternated between MS and data-dependent $\mathrm{MS}^{n}$ scans using dynamic exclusion. The scan range varied slightly between methods but covered $70-1000 \mathrm{~m} / \mathrm{z}$. Raw data files were archived and extracted as described below.

All time-course samples from each infant were assayed in the same analytic run. Quality control of the assay was monitored using four types of standards. The median relative standard deviation for instrument variability using internal standards was $4 \%$ and total process variability using endogenous biochemicals was $7 \%$.

\section{Statistical analysis}

The dataset comprised a total of 829 compounds of known identity (named biochemicals or metabolites). Raw area counts for each biochemical were obtained and adjusted for any inter-assay variability to generate Original Scale data. These values were then 
rescaled setting the median value to equal 1 and normalized to hematocrit (mean/sd 34.7 \pm 4.1 , range 26.6-40.0). Missing time point values (below the limit of detection), if any, were imputed using the minimum observed value for each compound (termed Scaled Imputed Data). Log-transformed Scaled Imputed Data were analyzed by principal component analysis and by two-way repeated measures analysis of variance to identify effects of budesonide by time (at 24 -h intervals), by dose $(0.025,0.05$, and $0.1 \mathrm{mg} / \mathrm{kg}$ ), and by interaction between dose and time. The false discovery rate ( $q$ value) was calculated to take into account the multiple comparisons that occur in untargeted metabolomics. ${ }^{13}$ In presentation of biochemical responses to budesonide treatment in this discovery analysis, we used a $q$ value $\leq 0.1$ that occurred for either time and/or dose:time interaction (referred to as "regulated metabolites"). The distribution of regulated metabolites by subpathway category was analyzed by chi-square and false discovery rate. To estimate the maximal fold change in metabolite level with budesonide treatment, we used Original Scale data and determined the ratio of the plateau response value (mean of values at 48,72 , and $96 \mathrm{~h}$ ) compared to the initial time point value (baseline $-15 \mathrm{~min}$ and/or $1 \mathrm{~h}$ ). Levels were similar at $15 \mathrm{~min}$ and $1 \mathrm{~h}$ for regulated metabolites that later decreased with treatment (ratio 1 $\mathrm{h} / 15 \mathrm{~min} 0.97 \pm 0.21, p=0.42$ ) and those that increased with treatment (ratio $1 \mathrm{~h} / 15 \mathrm{~min} 1.03 \pm 0.32, p=0.23$ ). The significance of the fold change was assessed using a paired $t$ test for all infants in each dose group comparing mean Original Scale data at plateau vs baseline. We used linear regression analysis to test the relationship between baseline TA IL-8 (log value) and each hormonally responsive $(n=90)$ blood biochemical. For these 90 biochemicals, we also assessed the pattern of dose response using the fold change and $p$ values at each dose level to assign a category of dose response pattern. We tested for effects of clinical variables on the fold change with budesonide treatment by linear regression (gestational age, birth weight, age at first dose, RSS at first dose, and days on parenteral nutrition) and by unpaired $t$ test (sex, race/ethnicity).

\section{RESULTS}

Metabolomics analysis used 121 blood samples that were serially collected during intra-tracheal budesonide in surfactant treatment of 23 infants in the SASSIE trial. Demographics and dosing data have been reported. ${ }^{11}$ For the entire group, gestational age was $25.0 \pm 1.3$ weeks; birth weight $743 \pm 204 \mathrm{~g}$; RSS at entry $3.4 \pm 1.5$ with $50 \%$ male; and race/ethnicity distribution of 12 White-nonHispanic, 7 White-Hispanic, 2 Black, and 2 unknown. Dosing was started at $6.5 \pm 3$ days with a mean of 4 (range 2-5) doses given at 24-h intervals. Metabolomics data were obtained from 8 infants at dose level 1 ( $0.025 \mathrm{mg}$ budesonide/ $\mathrm{kg}), 8$ at dose level $2(0.05 \mathrm{mg} /$ $\mathrm{kg})$, and 7 at dose level $3(0.1 \mathrm{mg} / \mathrm{kg})$.

By principle component analysis, the first two principal components explain 12 and $10 \%$ of the variance over all biochemicals, respectively. There was no shift in the global metabolic profile by budesonide dose or time point, which is consistent with selected effects of budesonide treatment on a subset of biochemicals (Fig. 1).

\section{Fold change}

Table 1 lists the 90 metabolites, of the 829 detected (10.9\%), that varied over time at $\geq 1$ budesonide doses with a $q$ value $\leq 0.1$. The maximum fold change with treatment was assessed by the ratio of the mean metabolite value at 48-96 h (defined as plateau response) compared to the mean of values at $15 \mathrm{~min}$ and $1 \mathrm{~h}$ (defined as baseline level). Fold change ranged from 1.14 (increase in 4hydroxyglutamate) to 8.3 (decrease in 21-hydroxypragnanolone monosulfate). The maximum fold change was similar for metabolites increased by budesonide $(1.74 \pm 0.5$-fold, $n=55)$ and those decreased (2.12 $\pm 1.54, p=0.10, n=35)$.
We used the fold change at $24 \mathrm{~h}$ vs the mean value at $15 \mathrm{~min}-1 \mathrm{~h}$ at dose level 3 to assess early kinetics of changes after exposure to the first dose of budesonide. For all 89 regulated metabolites with $24-\mathrm{h}$ data, the change at $24 \mathrm{~h}$ was $-2 \pm 37 \%$ (NS) for metabolites that were decreased by budesonide $(n=35)$ and $34 \pm$ $37 \%(n=54)$ for those increased by budesonide $(p<0.0001)$, indicating that, in general, blood levels for upregulated metabolites increased faster than the decrease for downregulated metabolites.

\section{Dose response}

Different dose-response patterns were observed for regulated metabolites. As illustrated in Fig. 2a for 1-palmitoyl-glycerol-3phosphoinositol, concentrations of some biochemicals did not change over time at dose level 1, increased significantly at dose level 2, and were higher at level 3 . In a second pattern as represented by $N$-palmitoyltaurine (Fig. 2b), there was a significant change at dose level 2 and a similar change at level 3 (consistent with a plateauing of response). Some metabolites had a significant change only at dose level 3 (Fig. 2c, phenylalanylalanine), indicating a relatively low sensitivity to budesonide. Another subset of metabolites demonstrated similar significant changes at all three dose levels consistent with high sensitivity to budesonide (Fig. 2d, pregnanediol-3-glucuronide). Other metabolites (Fig. 2e, methylphosphate) reached significance at dose level 2 but not at dose 1 or 3, which could reflect inter-infant variability or, possibly in some cases, a biphasic response. For comparison, Fig. $2 f$ shows the dose-response data for glycine, which did not respond to budesonide exposure.

Table 2 summarizes the direction of change for metabolites by the pattern of responsiveness (as listed in Table 2). The category of progressive change over the 3 dose levels (row 1) was composed primarily of metabolites that were increased by budesonide, and this represented a significant difference from the overall distribution of increased vs decreased biochemical $(55: 35, p=0.005)$. Of the 15 metabolites that were similarly responsive at each dose (row 4, All), 14 were decreased by budesonide exposure ( $p=0.015$ vs overall distribution). Overall, these results indicate variable sensitivity of blood biochemicals to budesonide with highest sensitivity for a subset of negatively regulated metabolites.

To examine possible influences of clinical variables on the response to budesonide, we analyzed the 15 metabolites with a similar response at each dose (row 4, All of Table 2), which included all infants and provided greater statistical power. There was no effect $(p>0.05)$ on fold change in metabolites by gestational age, birth weight, sex, race/ethnicity (White vs Hispanic), age at first dose, and RSS. For the variable of time receiving parenteral nutrition, the budesonide-induced increase in pentothenate (vitamin B5) was less with increasing days $(r=0.5$, $p=0.04$ ).

\section{Biological pathways}

Regulated metabolites were classified under 45 different subpathways (of 116 total) with 3-6-fold overrepresentation at $q<0.1$ for 4 sub-pathways (lysophospholipid, progestin steroids, endocannabinoid, and sphingolipid synthesis) of the Lipid super pathway and glutamate metabolism of the Amino Acid super pathway (Table 3 ). Of the 20 sub-pathways with $\geq 2$ regulated metabolites, the direction of change with budesonide exposure was the same for each metabolite in the sub-pathway in 15 cases. The most notable impact of budesonide was observed for lipid metabolism with increased concentrations in categories of phosphatidylcholine, lysophospholipid, long/medium/short-chain fatty acids, acyl carnitine, monohydroxy fatty acid, and phosphatidylserine and decreases for sphingolipid synthesis and sphingosines. Also, there were marked decreases $(2.2-8.3-\mathrm{fold})$ in concentrations of 8 steroids in categories of progestin, 


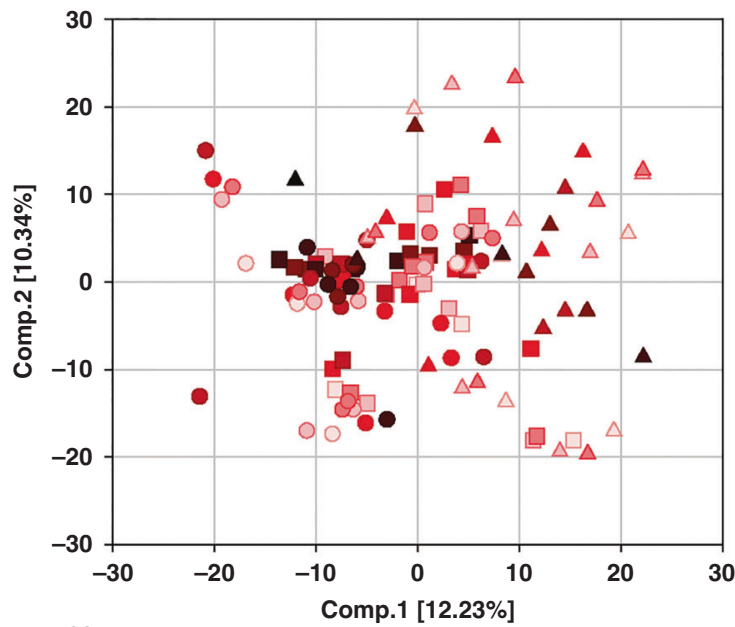

PCA Group:

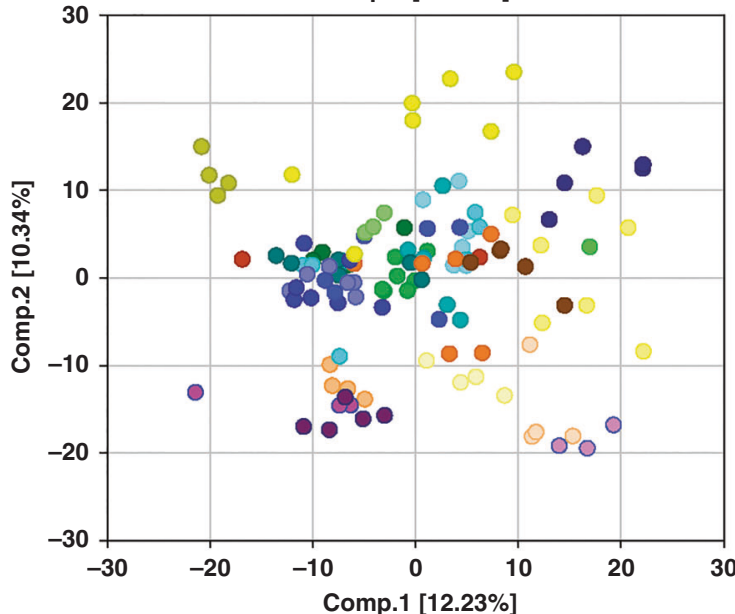

\begin{tabular}{|c|c|}
\hline $0.025 \mathrm{mg} / \mathrm{kg}$ & Infant \\
\hline $0.25 \mathrm{~h}$ & 1006 \\
\hline $1 \mathrm{~h}$ & 1015 \\
\hline $4 \mathrm{~h}$ & 1020 \\
\hline & 1021 \\
\hline $24 \mathrm{~h}$ & $\mid 1032$ \\
\hline $48 \mathrm{~h}$ & 1036 \\
\hline $72 \mathrm{~h}$ & 1039 \\
\hline $96 \mathrm{~h}$ & 3034 \\
\hline & 4025 \\
\hline $0.05 \mathrm{mg} / \mathrm{kg}$ & 4055 \\
\hline $0.25 \mathrm{~h}$ & 4056 \\
\hline $1 \mathrm{~h}$ & 5017 \\
\hline $4 \mathrm{~h}$ & 5025 \\
\hline & 5026 \\
\hline $24 \mathrm{n}$ & 5028 \\
\hline $48 \mathrm{~h}$ & 5029 \\
\hline $72 \mathrm{~h}$ & 5031 \\
\hline $96 \mathrm{~h}$ & 5038 \\
\hline & 5059 \\
\hline $0.1 \mathrm{mg} / \mathrm{kg}$ & 5061 \\
\hline & 5084 \\
\hline $0.25 \mathrm{~h}$ & 5088 \\
\hline$\triangle 1 \mathrm{~h}$ & 6002 \\
\hline$\triangle 4 h$ & 6011 \\
\hline$\triangle 24 h$ & Dose \\
\hline$\triangle 48 \mathrm{~h}$ & \\
\hline$\triangle 72 \mathrm{~h}$ & $0.025 \mathrm{mg} / \mathrm{kg}$ \\
\hline$\triangle 96 \mathrm{~h}$ & $0.05 \mathrm{mg} / \mathrm{kg}$ \\
\hline & $0.1 \mathrm{mg} / \mathrm{kg}$ \\
\hline
\end{tabular}

Fig. 1 Principal component analysis (PCA) of metabolomics data. The first two principal components explain 12 and $10 \%$ of the variance over all biochemicals, respectively. The analysis reveals that there is no global shift in metabolomics profile by dose (shape of data point) or time point (color intensity) (top plot) but rather that samples from the same infant are more similar to each other (bottom plot).

pregnenolone, estrogenic, and corticoid, consistent with suppression of adrenal steroidogenesis by glucocorticoid treatment. In the Amino Acid super pathway, in addition to metabolites related to glutamate metabolism, levels of 11 other amino acids were influenced by budesonide, with a similar number increased and decreased. There were two regulated vitamins, B1 decreased and B5 increased with budesonide, and only two regulated metabolites in the Carbohydrate super pathway that both decreased $(\mathrm{N}$-acetylglucosamine/ $\mathrm{N}$-acetylgalactosamine and ribulonate/xylulonate/lyxonate).

Relationship to inflammation

Budesonide and other corticosteroids are administered to infants with respiratory failure primarily for anti-inflammatory effects. Using blood samples collected at baseline (mean 15 min and $1 \mathrm{~h}$ ), we examined budesonide-regulated blood biochemicals for associations with TA IL-8, a measure of lung inflammatory status. ${ }^{11,14}$ For 20 infants with both IL- 8 and metabolite baseline data, 24 of the 90 regulated metabolites had a regression slope $p$ value of $<0.05$ (range $0.00048-0.044$ ), $q$ values $\leq 0.15$, and $r$ values ranging from 0.34 to $0.74-$ Table $1 ; 15$ metabolites had a positive slope and 9 had a negative slope related to IL-8. In 22 of the 24 instances, the direction of response with budesonide was in the opposite direction to that observed with increasing IL-8, and we designate these biochemicals as inflammation associated. As examples, levels of blood sphingosine increased with higher aspirate IL-8 ( $r=0.74, q=0.04$, Fig. 3a) and blood levels decreased by $40 \%$ with budesonide exposure (Table 1 ); blood levels of 1-palmitoyl glycerol-3-phosphocholine decreased with higher IL-8 (Fig. 3b) and increased with budesonide. Overall, the 22 inflammation-associated biochemicals were predominantly lipids (15), with 3 amino acids, 2 nucleotides, 1 vitamin (B1), and 1 glycolysis metabolite; all of the 4 dose-response patterns were represented.

\section{DISCUSSION}

In the SASSIE trial, increasing dose levels of budesonide in surfactant were administered to premature infants in respiratory failure to suppress lung inflammation and slow progression of injury and disease. We found that infants with elevated baseline levels of TA cytokines had a decrease in cytokines during treatment, which was independent of the budesonide dose. ${ }^{11}$ In the current report, we analyzed in detail the effect of budesonide on global blood metabolomics to assess systemic responses to intrapulmonary therapy. We describe a subset of blood biochemicals that are altered by treatment and that respond with apparent different sensitivities to budesonide. Levels of a subset of these regulated metabolites are associated with lung inflammatory status, representing potential blood biomarkers for infant lung inflammation. Overall, these novel findings define the systemic metabolic response to corticosteroid in premature infants and provide new information related to optimizing postnatal corticosteroid therapy. 
Table 1. Blood metabolites regulated by budesonide at $q \leq 0.1$.

\begin{tabular}{|c|c|c|c|c|c|c|c|c|}
\hline \multirow[t]{2}{*}{ Biochemical } & \multicolumn{3}{|c|}{ Maximum fold change } & \multirow[t]{2}{*}{ Dose response pattern } & \multicolumn{4}{|c|}{ Baseline level } \\
\hline & Mean & Sd & $\begin{array}{l}\text { FDR } \\
q\end{array}$ & & $r$ & $p$ & $q$ & Slope \\
\hline $\mathrm{N}$-acetyl-3-methylhistidine & 0.40 & 0.22 & $<0.0001$ & $A$ & 0.22 & 0.30 & 0.458 & \\
\hline Creatine & 0.79 & 0.11 & $<0.0001$ & A & 0.15 & 0.66 & 0.808 & \\
\hline Myristate (14:0) & 1.72 & 0.57 & $<0.0001$ & $\mathrm{~L}$ & 0.10 & 0.70 & 0.808 & \\
\hline 5Alpha-pregnan-diol disulfate & 0.48 & 0.20 & $<0.0001$ & $A$ & 0.05 & 0.85 & 0.932 & \\
\hline Pregnanediol-3-glucuronide & 0.28 & 0.18 & $<0.0001$ & A & 0.25 & 0.35 & 0.500 & \\
\hline Estriol 3-sulfate & 0.21 & 0.07 & $<0.0001$ & A & 0.26 & 0.30 & 0.458 & \\
\hline Taurolithocholate 3-sulfate & 0.76 & 0.43 & $<0.0001$ & A & 0.13 & 0.62 & 0.786 & \\
\hline Theophylline & 1.60 & 0.28 & $<0.0001$ & $\mathrm{~L}$ & 0.19 & 0.43 & 0.605 & \\
\hline Methyl vanillate sulfate & 2.30 & 1.63 & $<0.0001$ & $\mathrm{~L}$ & 0.10 & 0.70 & 0.808 & \\
\hline Kynurenate & 0.70 & 0.11 & 0.0005 & $\mathrm{~L}$ & 0.57 & 0.01 & 0.110 & 1 \\
\hline Sphingosine & 0.61 & 0.15 & 0.0005 & $\mathrm{O}$ & 0.74 & 0.0005 & 0.043 & 1 \\
\hline 1-Linoleoyl-GPC (18:2) & 3.12 & 1.91 & 0.0007 & $\mathrm{~L}$ & 0.37 & 0.13 & 0.266 & \\
\hline $\mathrm{N}$-acetylglucosamine $/ \mathrm{N}$-acetylgalactosamine & 0.74 & 0.11 & 0.0008 & A & 0.43 & 0.072 & 0.196 & \\
\hline 1-Docosahexaenoylglycerol (22:6) & 0.75 & 0.18 & 0.0008 & $\mathrm{O}$ & 0.56 & 0.017 & 0.121 & I \\
\hline 1-Palmitoyl-GPC (16:0) & 1.69 & 0.55 & 0.0009 & $\mathrm{~L}$ & 0.62 & 0.006 & 0.108 & $\mathrm{D}$ \\
\hline 1-Linolenoyl-GPC (18:3) & 1.76 & 0.47 & 0.0010 & $\mathrm{O}$ & 0.55 & 0.021 & 0.121 & $\mathrm{D}$ \\
\hline Quinolinate & 0.71 & 0.09 & 0.0012 & $\mathrm{D} 2$ & 0.23 & 0.35 & 0.500 & \\
\hline Ribulonate/xylulonate/lyxonate & 0.62 & 0.24 & 0.0015 & A & 0.44 & 0.066 & 0.196 & \\
\hline 2-Palmitoyl-GPC (16:0) & 1.71 & 0.64 & 0.0015 & $\mathrm{~L}$ & 0.42 & 0.081 & 0.203 & \\
\hline 1-Stearoyl-GPI (18:0) & 1.31 & 0.58 & 0.0037 & $\mathrm{D} 2$ & 0.33 & 0.18 & 0.324 & \\
\hline Pantothenate & 1.30 & 0.13 & 0.0049 & A & 0.03 & 0.91 & 0.964 & \\
\hline S-(3-hydroxypropyl)mercapturic acid (HPMA) & 0.75 & 0.20 & 0.0052 & $\mathrm{~L}$ & 0.42 & 0.08 & 0.203 & \\
\hline Cortisone & 0.49 & 0.26 & 0.0055 & A & 0.39 & 0.11 & 0.254 & \\
\hline 21-Hydroxypregnanolone monosulfate (1) & 0.12 & 0.08 & 0.0060 & A & 0.60 & 0.024 & 0.121 & 1 \\
\hline$N$-palmitoyltaurine & 1.62 & 0.28 & 0.0061 & $\mathrm{D} 2$ & 0.34 & 0.16 & 0.300 & \\
\hline Eicosanedioate (C20-DC) & 1.23 & 0.15 & 0.0062 & $\mathrm{O}$ & 0.41 & 0.04 & 0.150 & $\mathrm{D}$ \\
\hline Sphinganine & 0.60 & 0.23 & 0.0081 & $\mathrm{O}$ & 0.66 & 0.005 & 0.108 & 1 \\
\hline Behenate $(22: 0)$ & 1.16 & 0.10 & 0.0096 & $\mathrm{O}$ & 0.37 & 0.13 & 0.266 & \\
\hline 3-(4-Hydroxyphenyl)lactate & 0.43 & 0.16 & 0.0098 & $\mathrm{O}$ & 0.51 & 0.03 & 0.121 & 1 \\
\hline Caprate (10:0) & 1.94 & 1.11 & 0.0098 & $\mathrm{~L}$ & 0.16 & 0.52 & 0.678 & \\
\hline Docosadioate (C22-DC) & 1.39 & 0.19 & 0.0102 & $\mathrm{~L}$ & 0.51 & 0.031 & 0.121 & $\mathrm{D}$ \\
\hline 1-Oleoyl-2-docosahexaenoyl-GPC (18:1/22:6) & 0.79 & 0.10 & 0.0102 & $\mathrm{O}$ & 0.59 & 0.009 & 0.110 & 1 \\
\hline $\mathrm{N}$-acetylasparagine & 1.36 & 0.13 & 0.0110 & $\mathrm{~L}$ & 0.34 & 0.01 & 0.110 & $\mathrm{D}$ \\
\hline 1-Myristoyl-2-palmitoyl-GPC (14:0/16:0) & 1.56 & 0.48 & 0.0136 & $\mathrm{D} 2$ & 0.41 & 0.088 & 0.214 & \\
\hline 2-Hydroxypalmitate & 1.17 & 0.14 & 0.0163 & $\mathrm{~L}$ & 0.34 & 0.16 & 0.300 & \\
\hline CoA-glutathione & 2.20 & 1.12 & 0.0171 & $\mathrm{O}$ & 0.41 & 0.12 & 0.266 & \\
\hline Hydroquinone sulfate & 2.73 & 2.00 & 0.0171 & $\mathrm{~L}$ & 0.30 & 0.23 & 0.383 & \\
\hline 1-Palmitoleoyl-GPC (16:1) & 1.48 & 0.91 & 0.0179 & $\mathrm{~L}$ & 0.23 & 0.35 & 0.500 & \\
\hline Gamma-glutamylglutamine & 2.56 & 1.44 & 0.0181 & $\mathrm{~L}$ & 0.42 & 0.87 & 0.932 & \\
\hline
\end{tabular}




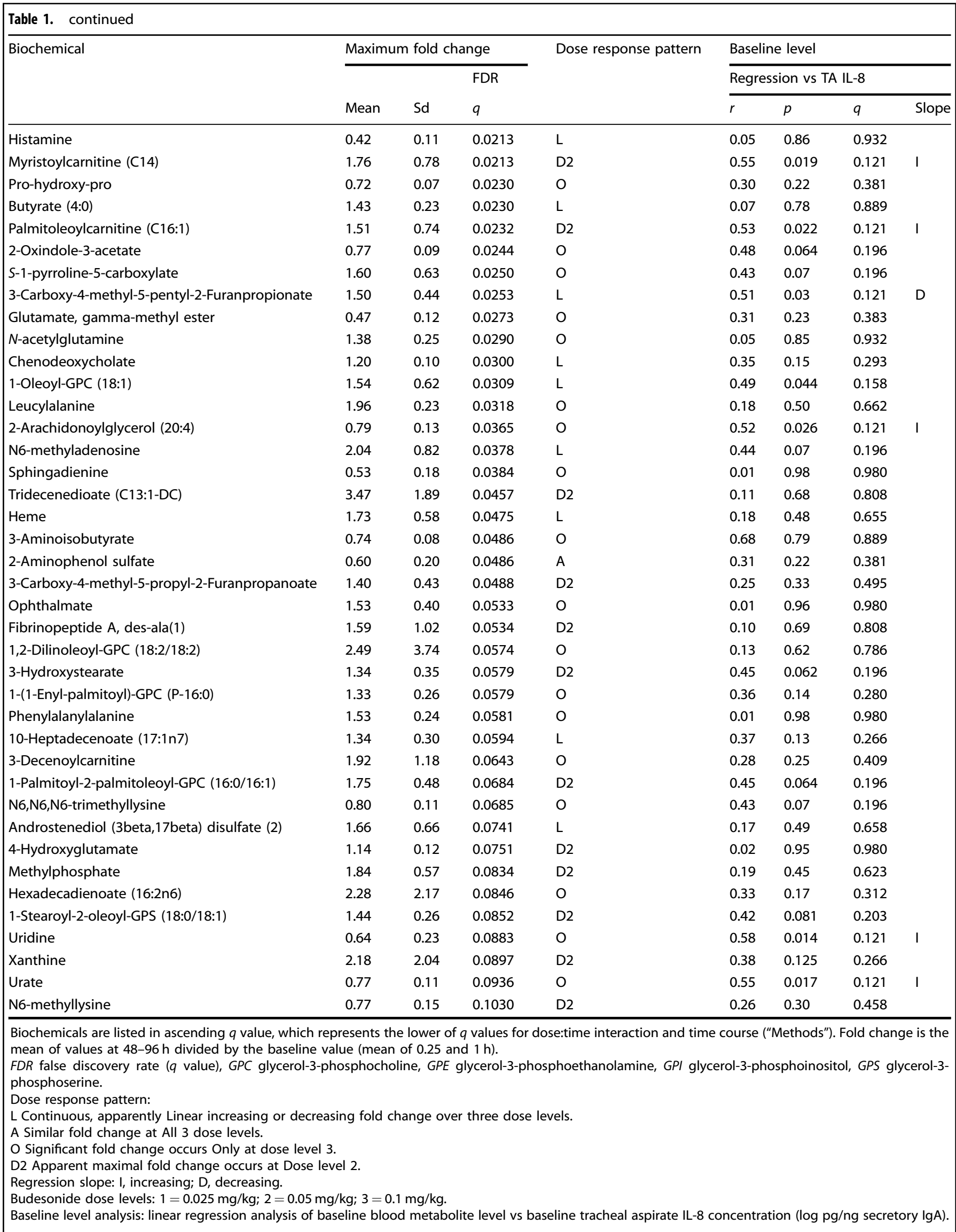


Table 2. Pattern of dose response to budesonide and direction of change.

\begin{tabular}{llll} 
Dose response pattern & Number of metabolites & \\
\cline { 2 - 4 } & Increase in concentration & Decrease in concentration \\
\hline Progressive change over 3 dose levels (Linear) & 26 & 3 & 0.005 \\
Apparent maximal change at dose level 2 (Dose 2) & 14 & 4 & 0.18 \\
Change occurs only at dose level 3 (Only) & 14 & 14 & 0.30 \\
Similar change all 3 dose levels (All) & 1 & 14 & 0.015 \\
\hline
\end{tabular}

Data are for 90 metabolites listed in Table 1. Abbreviation for response pattern as noted in Table 1.

$p$, significance by chi-squared compared to distribution of all increased (55) and decreased (35) metabolites.
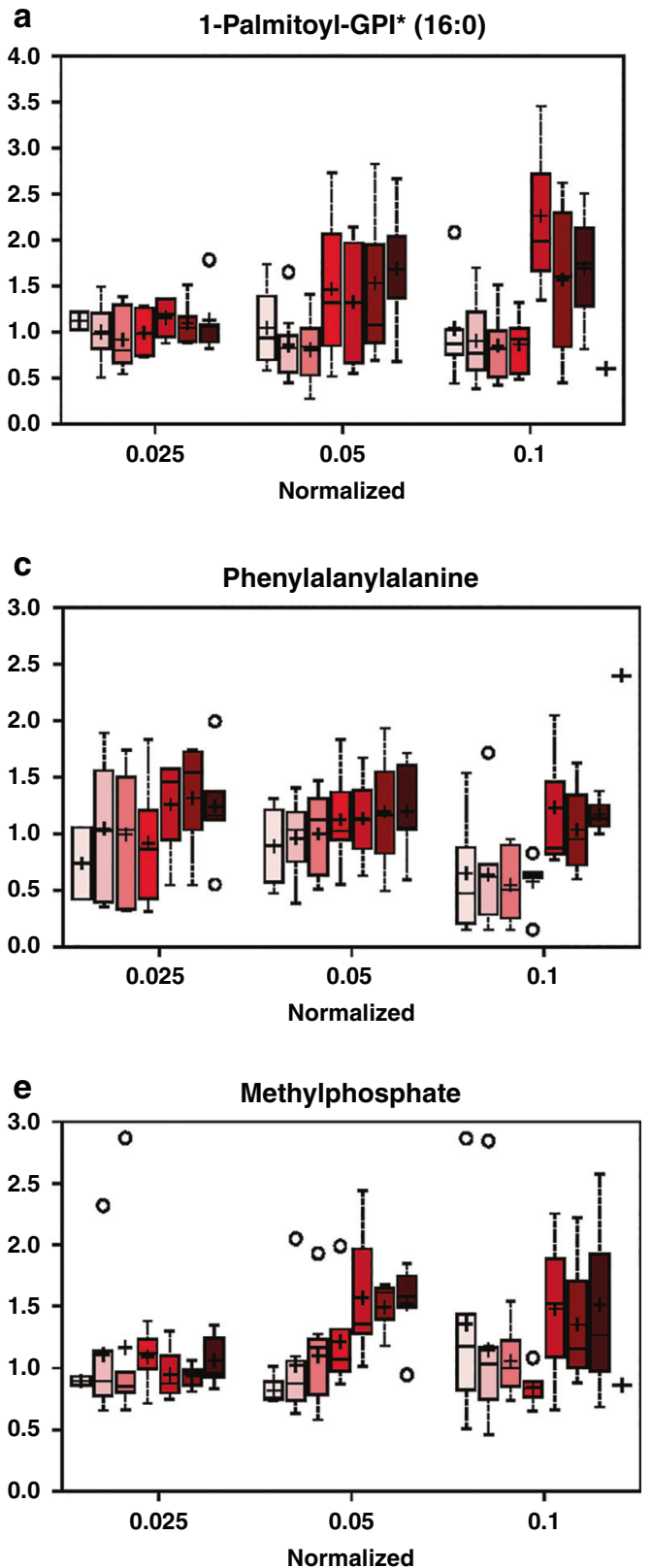
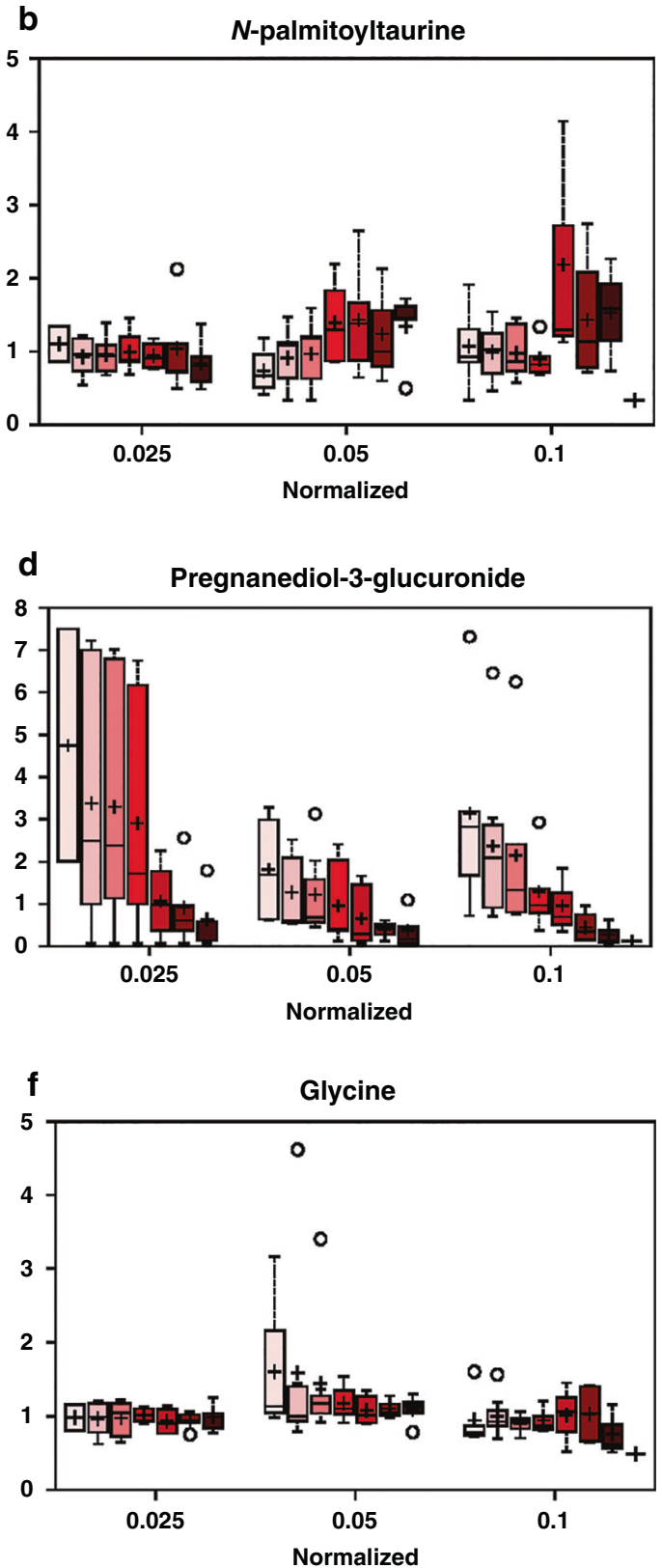

Fig. 2 Time:dose plots representing examples of different patterns of response to daily budesonide treatment. a Increasing response over 3 doses; $\mathbf{b}$ apparent plateau response at dose level $2(0.05 \mathrm{mg} / \mathrm{kg}) ; \mathbf{c}$ significant response only at highest dose; $\mathbf{d}$ similar response at all three doses; e, significant response only at dose level 2; f no significant response at any dose. Boxes for each dose are (left to right) $15 \mathrm{~min}, 1 \mathrm{~h}, 4 \mathrm{~h}$, $24 \mathrm{~h}, 48 \mathrm{~h}, 72 \mathrm{~h}$, and $96 \mathrm{~h}$ after first treatment. Data are normalized to median value ("Methods"). *Identity not confirmed with standard but high confidence per metabolon. 
Table 3. Sub-pathway designation and relative representation of metabolites regulated by budesonide at $q \leq 0.10$.

\begin{tabular}{|c|c|c|c|c|c|}
\hline \multirow[t]{2}{*}{ Sub-pathway } & \multirow{2}{*}{$\begin{array}{l}\text { Regulated } \\
\text { metabolites } \\
n\end{array}$} & \multirow{2}{*}{$\begin{array}{l}\text { Total detected } \\
\text { metabolites } \\
n\end{array}$} & \multirow{2}{*}{$\begin{array}{l}\text { Regulated/total } \\
\%\end{array}$} & \multirow{2}{*}{$\begin{array}{l}\text { Fold change } \\
\text { Mean } \pm \text { sd }\end{array}$} & \multirow{2}{*}{$\begin{array}{l}\text { Compare vs All } \\
q\end{array}$} \\
\hline & & & & & \\
\hline Purine and pyrimidine metabolism ${ }^{a}$ & 6 & 51 & 11.8 & 3 inc, 3 dec & 0.91 \\
\hline Fatty acid, dicarboxylate & 5 & 24 & 20.8 & $1.80 \pm 0.94$ & 0.33 \\
\hline Glutamate metabolism & 4 & 11 & 36.4 & 3 inc, 1 dec & 0.035 \\
\hline Phosphatidylcholine (PC) & 4 & 15 & 26.7 & 3 inc, 1 dec & 0.17 \\
\hline $\begin{array}{l}\text { Fatty acid metabolism (acyl carnitine, long- } \\
\text { chain sat.) }\end{array}$ & 3 & 42 & 7.1 & $1.51 \pm .41$ & 0.66 \\
\hline Progestin steroids & 3 & 5 & 60.0 & $0.35 \pm 0.11$ & 0.004 \\
\hline Androgenic steroids & 2 & 8 & 25.0 & $0.45,1.66$ & 0.39 \\
\hline Chemical & 2 & 18 & 11.1 & $0.60,0.75$ & 0.96 \\
\hline Food component/plant & 2 & 32 & 6.3 & $0.77,2.30$ & 0.65 \\
\hline Glutathione metabolism & 2 & 11 & 18.2 & $1.53,1.68$ & 0.66 \\
\hline Histidine metabolism & 2 & 18 & 11.1 & $0.40,0.42$ & 0.96 \\
\hline Lysine metabolism & 2 & 16 & 12.5 & $0.77,0.80$ & 0.91 \\
\hline Medium-chain fatty acid & 2 & 7 & 28.6 & $1.34,1.94$ & 0.33 \\
\hline Monoacylglycerol & 2 & 16 & 12.5 & $0.72,0.79$ & 0.91 \\
\hline Sphingolipid synthesis & 2 & 3 & 66.7 & $0.53,0.60$ & 0.013 \\
\hline \multicolumn{6}{|c|}{$\begin{array}{l}\text { Total detected metabolites were } 829 \text { with } 90 \text { regulated at } q \leq 0.10(11.0 \%) \text {, representing } 47 \text { different sub-pathways. No designated sub-pathway for one } \\
\text { regulated metabolite (hydroquinone sulfate). } \\
\text { Fold change is mean value at } 48-96 \mathrm{~h} \text { divided by mean of } 15 \mathrm{~min} \text { and } 1 \mathrm{~h} \text { values. Chi-squared and False discovery rate analyses were used for assigning } q \\
\text { values for the number of regulated metabolites in sub-pathways with }>1 \text { regulated metabolite compared to the overall total regulated metabolites (Compare } \\
\text { vs All). Five sub-pathways were overrepresented at } q \leq 0.1 \text { for regulated biochemicals. } \\
\text { inc increase, dec decrease. } \\
\text { a Purine and pyrimidine metabolism plus purine metabolism, (hypo)xanthine/inosine containing plus purine metabolism, adenine containing plus pyrimidine } \\
\text { metabolism, and uracil containing. } \\
\text { b Long-chain monounsaturated fatty acid plus long-chain polyunsaturated fatty acid (n3 and n6) plus long-chain saturated fatty acid. }\end{array}$} \\
\hline
\end{tabular}

There are limited published data for metabolomics responses to glucocorticoid exposure in children and adults. Bordag et al. ${ }^{15}$ studied adult plasma samples after exposure to dexamethasone; 2 of the 7 species of lysophospholipid increased, similar to our observations (9 of 26). Quan-Jun et al. ${ }^{16}$ compared serum metabolites in asthmatic children receiving inhaled budesonide plus salbutamol vs an untreated group. Of the 22 observed metabolite differences, the arginine and proline pathways were most highly represented; in comparison with our findings, there was agreement with regard to decreased levels of creatine and histamine with treatment.

In a previous study of metabolic markers of infant lung disease, La Frano et al. $^{9}$ found decreased levels of phosphatidylcholines and sphingomyelins in cord blood associated with later development of pulmonary hypertension. There is one previous report addressing untargeted pharmacometabolomics effects of corticosteroid treatment of premature infants. ${ }^{7}$ Serum samples were collected before and after systemic dexamethasone given to ten infants for respiratory failure. Of the 17 identified metabolites where levels changed with treatment, 13 were also detected in our study. We found no significant change for 12 of these metabolites and a significant increase for 1 (pantothenate), which is in the opposite direction than reported by Lewis et al. (fourfold decrease). We also detected no change in the levels of gluconic acid in contrast to $>100$-fold decrease reported. It is possible that the differing results reflect an effect of postnatal age (26-127 vs
3-12 days for SASSIE infants), type of nutrition, serum vs whole blood, systemic dexamethasone vs instilled budesonide, and/or assay techniques. Replication studies will be required to address the different findings.

We report the new observation, to our knowledge, of variable time and dose response characteristics for metabolic effects of glucocorticoid in the humans. Because instilled budesonide is cleared from blood with a half-life of $3.4 \mathrm{~h},{ }^{11,17}$ daily treatment, as we used, represents a consistent peak concentration and distinct corticosteroid exposure at each dose level, i.e., peak blood budesonide levels do not increase with daily treatment. ${ }^{11,17}$ Glucocorticoids have three major areas of biological action: accelerated maturation of fetal tissues, regulation of tissue metabolism for homeostasis and adaptive responses, and antiinflammatory/immune-suppression effects. Most maturational and metabolic actions occur by interaction of the glucocorticoid receptor complex at positive or negative glucocorticoid response elements of target genes (direct genomic mechanism). Some immunosuppressive/anti-inflammatory actions involve a direct genomic mechanism, but others occur via interaction of glucocorticoid receptor with other transcription factors (e.g., nuclear factor (NF)-KB-indirect genomic mechanism) and by a non-genomic mechanism involving a cytoplasmic second messenger cascade. Variable kinetics and dose responsiveness could arise because of different properties of glucocorticoid response elements, affinity of other transcription factors for their multiple 

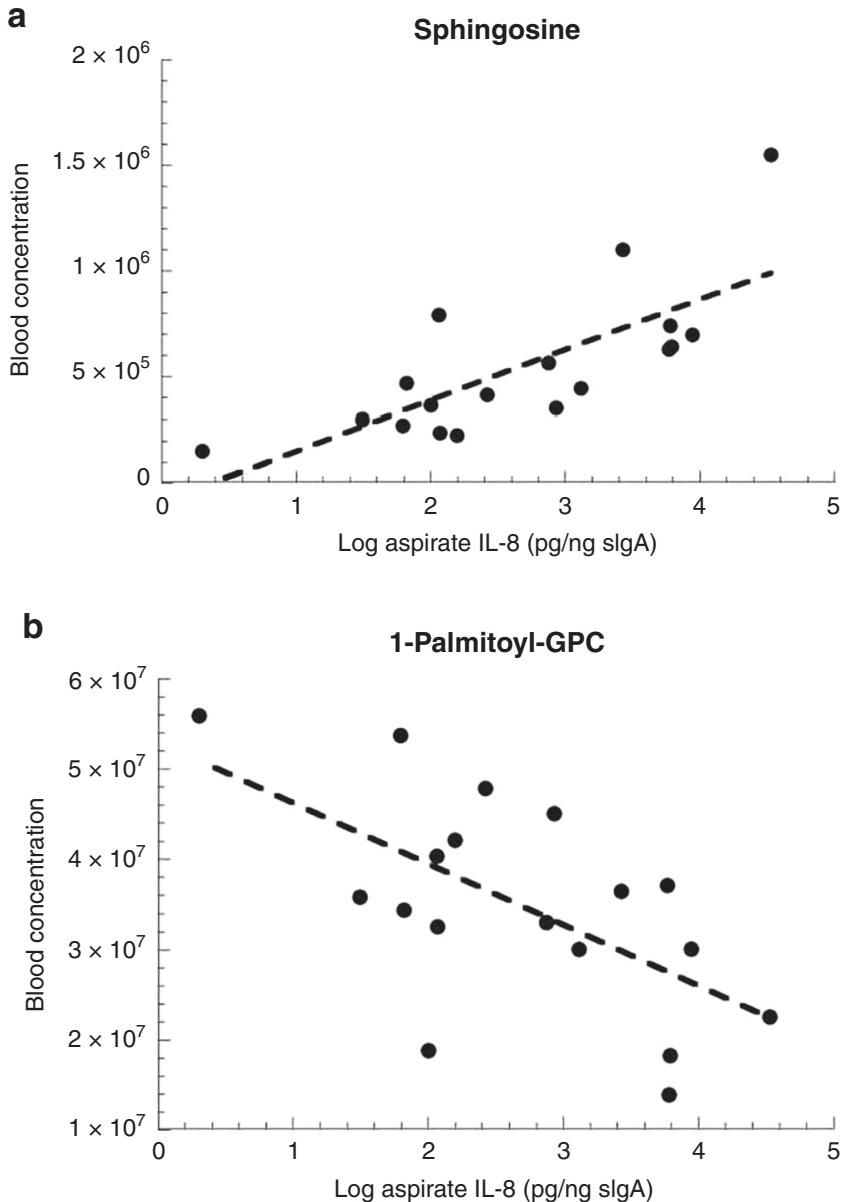

Fig. 3 Relationship at baseline of selected blood metabolite levels to IL-8 in tracheal aspirate. a Sphingosine: $r=0.74, p=0.0005$. b, 1Palmitoyl-glycerol-3-phosphocholine: $\quad r=0.62, \quad p=0.006 . \quad N=20$ infants.

response elements, and rapidity of non-genomic vs genomic mechanisms. ${ }^{18}$ Thus it is not surprising that we observed different time and dose responses for blood metabolites as downstream responses of enhanced or suppressed enzyme activity with budesonide treatment. All of the observed responses are consistent with a genomic mechanism as there were no examples of a rapid change $(\leq 4 \mathrm{~h})$ in metabolite level. We speculate that the 14 biochemicals that were maximally repressed at the lowest budesonide dose reflect glucocorticoid interaction with NF-KB; previously, we found that inhibition of cytokines in cultured fetal lung, which is mediated via NF-KB, occurred at a lower budesonide concentration than for induction of surfactant proteins. ${ }^{19}$ These metabolites (e.g., estriol 3-sulfate) represent potential systemic biomarkers with high sensitivity to glucocorticoid exposure in the premature infant.

While additional studies are needed to confirm the inflammation-related status of selected budesonide-regulated biochemicals, there is published information and rationale for an influence of inflammation and/or glucocorticoid.

1. Sphingosine and precursor sphinganine. Candidate enzymes for regulation in the de novo synthetic pathway to ceramide, sphingomyelins, and sphingosine are serine palmitoyltransferase and dihydroceramide desaturase, which are both influenced by stress and glucocorticoids. $^{20-22}$ Lung injury due to hyperoxia, acute respiratory distress syndrome, and bleomycin is associated with increased sphingomyelins, ceramides, and sphingosine, suggesting that the recycling pathway is also affected by inflammation. ${ }^{23}$ Budesonide did not significantly $(p=0.1)$ decrease levels of sphingosine-1-phosphate, which is a mediator of inflammatory processes in asthma and other diseases. $^{24,25}$ A decrease in sphingosine with budesonide, acting in the recycling pathway, would be consistent with effects of both lung maturation and glucocorticoid treatment to reduce the sphingomyelin:phosphatidylcholine ratio of amniotic fluid. ${ }^{23}$

2. Lysophopholipids and fatty acid dicarboxylic acids. Findings for these lipids are in agreement with results for lysophophatidylcholines and mortality in critically ill adults ${ }^{26}$ and increased lipolysis as one component of glucocorticoidinduced hyperglycemia. $^{18}$

3. Metabolites of asparagine/alanine ( $N$-acetylasparagine), tryptophan (kynurenate), and tyrosine (3-(4-hydroxyphenyl) lactate). The tryptophan to NAD pathway is upregulated in conditions with immune activation and both intermediates have neurotoxic properties related to inflammation and oxidative stress. ${ }^{27,28}$ Also, increased levels of kynurenate were previously observed for critically ill adults with lung inflammation/disease. ${ }^{26}$ Tryptophan metabolism could involve tryptophan 2,3-dioxygenase and/or indolamine 2,3dioxygenase, which are key enzymes that are regulated by both cytokines and glucocorticoids. ${ }^{29}$

4. Thiamin (vitamin B1). Thiamin is a cofactor for enzymes of glucose metabolism, acting through its diphosphorylated form (TPP), and the demand for TPP increases with high

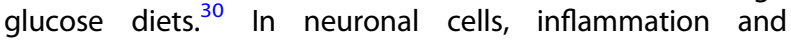
oxidative stress decrease thiamin metabolism and functions, which results in increased apoptosis. ${ }^{31}$ Premature infants with respiratory failure routinely receive intravenous multivitamins that include vitamin B1. It is possible that infant lung disease results in decreased tissue uptake of thiamin and/or phosphorylation, resulting in higher blood levels, and that glucocorticoid exposure reverses these effects.

5. Adrenal steroids 21-hydroxypregnanolone sulfate and androsteroid sulfate. Dehydroepiandrosterone-sulfate (DHEAS) is a product of the fetal zone of the adrenal, which remains active for many weeks after premature delivery, and circulating DHEAS is downregulated by both antenatal and postnatal glucocorticoid therapy ${ }^{32-34}$ acting via the hypothalamic-pituitary-adrenal (HPA) axis. The observed positive correlation of DHEAS with TA IL-8 could reflect stress activation of the HPA axis or stimulatory effects of inflammatory mediators on sulfatase/sulfotransferase activities resulting in increased ratio of DHEAS to DHEA such as observed in lipopolysaccharides-exposed macrophages, ${ }^{35}$ although other studies have found that cytokines increase sulfatase activity. ${ }^{36}$ DHEA, which is present in blood at many-fold lower concentrations than DHEAS, was not detected in our analyses.

6. Uridine and its metabolite uric acid. A decrease in uridine concentration with glucocorticoid treatment was also observed in adults. ${ }^{37}$ These findings are consistent with regulation at the level of de novo synthesis and may include diversion of uridine to lipid or sugar conjugates, which are required for liver glycogen deposition, and/or reduced ribose synthesis. Blood uridine levels could also decrease secondary to increased cellular uptake (transport pathway), which is stimulated by elevated amino acids and glucagon, and by enhanced biliary clearance as observed during refeeding. ${ }^{38-40}$

Although blood concentrations of histamine (and $\mathrm{N}$-acetyl-3methylhistamine) were not associated with lung IL-8, the 2.4 -fold 
suppression by budesonide may be relevant to beneficial effect of glucocorticoids for infants. Histamine is released from basophils and connective tissue mast cells and promotes bronchoconstriction, which is a component of lung disease in premature infants. The histamine response was optimal at $24 \mathrm{~h}$ and was observed only at the highest dose level, which is distinct from the rapid inhibition of histamine release from mast cells by high-dose corticosteroid. ${ }^{41}$

Some limitations of our pilot study should be noted. The number of infants studied was modest (total 23); thus the positive findings for candidate budesonide-regulated and inflammationassociated biochemicals will require validation. We reduced the additional experimental variability associated with DBS compared to blood samples by standardizing blood spot collection, storing DBS at $-20^{\circ} \mathrm{C}$ and normalizing results for hematocrit. ${ }^{42}$ It is known that the metabolomics profile is influenced by diet; however, all SASSIE infants received parenteral nutrition with lipid and amino acid formulations, with limited oral feedings, which is standard of care for intubated premature infants early in the postnatal course. Thus some of our results may apply only to White and Hispanic infants receiving parenteral nutrition. Also, we did not have a control (untreated) group and do not have access to a validation cohort for confirmation of key findings, which will be required before clinical studies evaluating the usefulness of a metabolic fingerprint can be performed. Finally, we did not include infants receiving the $0.25 \mathrm{mg} / \mathrm{kg}$ higher dose used by Yeh et al., ${ }^{5,6}$ based on the study protocol, which tested the hypothesis that lower doses of budesonide would be efficacious with lower systemic risk. ${ }^{11}$

Our combined results for lung inflammation ${ }^{11}$ and blood metabolomics suggest that optimal suppression of lung inflammation with limited systemic effects occurs at the lowest tested dose of budesonide $(0.025 \mathrm{mg} / \mathrm{kg})$ in surfactant. Because levels of budesonide with instillation are considerably higher within the lung than systemically, it is possible that even lower intra-tracheal doses provide effective anti-inflammatory treatment. There is the important caveat, however, that suppression of systemic inflammation and/or systemic metabolic effects, as occurs at higher doses, may contribute to a favorable clinical response in the lung. Ultimately, the optimal lung-targeted treatment regimen will require additional clinical trials that include measures of lung and systemic biochemical responses as well as pulmonary and neurodevelopmental outcomes.

\section{ACKNOWLEDGEMENTS}

We thank the parents, nurses, neonatal nurse practitioners, and respiratory therapists in the NICU for their support of the trial. We also appreciate the assistance of Theresa Rogers, RN and Steven Steele, RN, coordinators at Vanderbilt University Medical Center, and the time and expertise of the DSMB: Dr. Patricia Spitale (chair), Dr. Thuy Nguyen, and Dr. Eric Chen. This work was supported by Thrasher Research Fund (to C.T.M.) and NIH R21 HD097600 (to P.L.B., D.T.). ONY, Inc. provided Infasurf but was not involved in study design, collection, analysis or interpretation of data, writing the manuscript, or the decision to submit for publication.

\section{AUTHOR CONTRIBUTIONS}

Substantial contributions to trial conception and design, acquisition of data, analysis and interpretation of data, revising manuscript critically for important intellectual content, and final approval of the version to be published: all authors. Drafting the article: P.L.B.

\section{ADDITIONAL INFORMATION}

Competing interests: The authors declare no competing interests.

Consent: The research protocol was approved by the Institutional Review Boards of the participating institutions, and a parent of each infant provided written informed consent.
Publisher's note Springer Nature remains neutral with regard to jurisdictional claims in published maps and institutional affiliations.

\section{REFERENCES}

1. Jobe, A. H. \& Bancalari, E. Bronchopulmonary dysplasia. Am. J. Respir. Crit. Care Med. 163, 1723-1729 (2001).

2. Morrow, L. A. et al. Antenatal determinants of bronchopulmonary dysplasia and late respiratory disease in preterm infants. Am. J. Respir. Crit. Care Med. 196, 364-374 (2017)

3. Stoll, B. J. et al. Late-onset sepsis in very low birth weight neonates: the experience of the NICHD Neonatal Research Network. Pediatrics 110, 285-291 (2002).

4. Doyle, L. W., Cheong, J. L., Ehrenkranz, R. A. \& Halliday, H. L. Late (> 7 days) systemic postnatal corticosteroids for prevention of bronchopulmonary dysplasia in preterm infants. Cochrane Database Syst. Rev. 10, CD001145 (2017).

5. Yeh, T. F. et al. Early intratracheal instillation of budesonide using surfactant as a vehicle to prevent chronic lung disease in preterm infants: a pilot study. Pediatrics 121, e1310-e1318 (2008).

6. Yeh, T. F. et al. Intratracheal administration of budesonide/surfactant to prevent bronchopulmonary dysplasia. Am. J. Respir. Crit. Care Med. 193, 86-95 (2016).

7. Lewis, T., Chalise, P., Gauldin, C. \& Truog, W. Pharmacometabolomics of respiratory phenotypic response to dexamethasone in preterm infants at risk for bronchopulmonary dysplasia. Clin. Transl. Sci. 12, 591-599 (2019).

8. Clish, C. B. Metabolomics: an emerging but powerful tool for precision medicine. Cold Spring Harb. Mol. Case Stud. 1, a000588 (2015).

9. La Frano, M. R. et al. Umbilical cord blood metabolomics reveal distinct signatures of dyslipidemia prior to bronchopulmonary dysplasia and pulmonary hypertension. Am. J. Physiol. Lung Cell. Mol. Physiol. 315, L870-L881 (2018).

10. Stringer, K. A., McKay, R. T., Karnovsky, A., Quemerais, B. \& Lacy, P. Metabolomics and its application to acute lung diseases. Front. Immunol. 7, 44 (2016).

11. McEvoy, C. T. et al. Dose-escalation trial of budesonide in surfactant for prevention of bronchopulmonary dysplasia in extremely low gestational age highrisk newborns (SASSIE). Pediatr. Res. 88, 629-636 (2020).

12. Long, T. et al. Whole-genome sequencing identifies common-to-rare variants associated with human blood metabolites. Nat. Genet. 49, 568-578 (2017).

13. Benjamin, Y. \& Hochberg, Y. Controlling the false discovery rate: a practical and powerful approach to multiple testing. J. R. Stat. Soc B 57, 289-300 (1995).

14. Merrill, J. D. et al. Pilot trial of late booster doses of surfactant for ventilated premature infants. J. Perinatol. 31, 599-606 (2011).

15. Bordag, N. et al. Glucocorticoid (dexamethasone)-induced metabolome changes in healthy males suggest prediction of response and side effects. Sci. Rep. 5, 15954 (2015).

16. Quan-Jun, Y. et al. Distinct metabolic profile of inhaled budesonide and salbutamol in asthmatic children during acute exacerbation. Basic Clin. Pharmacol. Toxicol. 120, 303-311 (2017).

17. Rower, J. E. et al. Development and validation of an assay for quantifying budesonide in dried blood spots collected from extremely low gestational age neonates. J. Pharm. Biomed. Anal. 167, 7-14 (2019).

18. Rhen, T. \& Cidlowski, J. A. Antiinflammatory action of glucocorticoids-new mechanisms for old drugs. N. Engl. J. Med. 353, 1711-1723 (2005).

19. Barrette, A. M. et al. Antiinflammatory effects of budesonide in human fetal lung. Am. J. Respir. Cell Mol. Biol. 55, 623-632 (2016).

20. Linn, S. C. et al. Regulation of de novo sphingolipid biosynthesis and the toxic consequences of its disruption. Biochem. Soc. Trans. 29, 831-835 (2001).

21. Holland, W. L. et al. Inhibition of ceramide synthesis ameliorates glucocorticoid-, saturated-fat, and obesity-induced insulin resistance. Cell Metab. 5, 167-179 (2007)

22. Devlin, C. M. et al. Dihydroceramide-based response to hypoxia. J. Biol. Chem. 286, 38069-38078 (2011)

23. Ghidoni, R., Caretti, A. \& Signorelli, P. Role of sphingolipids in the pathobiology of lung inflammation. Mediators Inflamm. 2015, 487508 (2015).

24. Sukocheva, O. A., Lukina, E., McGowan, E. \& Bishayee, A. Sphingolipids as mediators of inflammation and novel therapeutic target in inflammatory bowel disease. Adv. Protein Chem. Struct. Biol. 120, 123-158 (2020).

25. Che, W. et al. Corticosteroids inhibit sphingosine 1-phosphate-induced interleukin-6 secretion from human airway smooth muscle via mitogenactivated protein kinase phosphatase 1-mediated repression of mitogen and stress-activated protein kinase 1. Am. J. Respir. Cell Mol. Biol. 50, 358-368 (2014).

26. Rogers, A. J. et al. Metabolomic derangements are associated with mortality in critically ill adult patients. PLOS ONE 9, e87538 (2014).

27. Chen, Y. \& Guillemin, G. J. Kynurenine pathway metabolites in humans: disease and healthy States. Int. J. Tryptophan Res. 2, 1-19 (2009).

28. Zinger, A., Barcia, C., Herrero, M. T. \& Guillemin, G. J. The involvement of neuroinflammation and kynurenine pathway in Parkinson's disease. Parkinsons Dis. 2011, 716859 (2011) 
Blood metabolomics in infants enrolled in a dose escalation pilot trial...

PL Ballard et al.

794

29. Badawy, A. A. Kynurenine pathway of tryptophan metabolism: regulatory and functional aspects. Int. J. Tryptophan Res. 10, 1178646917691938 (2017).

30. Lonsdale, D. A review of the biochemistry, metabolism and clinical benefits of thiamin(e) and its derivatives. Evid. Based Complement. Alternat. Med. 3, 49-59 (2006).

31. Chornyy, S., Parkhomenko, Y. \& Chorna, N. Thiamine antagonists trigger p53dependent apoptosis in differentiated SH-SY5Y cells. Sci. Rep. 7, 10632 (2017).

32. Heckmann, M. et al. Persistent high activity of the fetal adrenal cortex in preterm infants: is there a clinical significance? J. Pediatr. Endocrinol. Metab. 19, 1303-1312 (2006).

33. Ballard, P. L., Gluckman, P. D., Liggins, G. C., Kaplan, S. L. \& Grumbach, M. M. Steroid and growth hormone levels in premature infants after prenatal betamethasone therapy to prevent respiratory distress syndrome. Pediatr. Res. 14, 122-127 (1980).

34. Kari, M. A., Raivio, K. O., Stenman, U. H. \& Voutilainen, R. Serum cortisol, dehydroepiandrosterone sulfate, and steroid-binding globulins in preterm neonates: effect of gestational age and dexamethasone therapy. Pediatr. Res. 40, 319-324 (1996).
35. Schuld, A. et al. Changes in dehydroepiandrosterone (DHEA) and DHEA-sulfate plasma levels during experimental endotoxinemia in healthy volunteers. J. Clin. Endocrinol. Metab. 85, 4624-4629 (2000).

36. Mueller, J. W., Gilligan, L. C., Idkowiak, J., Arlt, W. \& Foster, P. A. The regulation of steroid action by sulfation and desulfation. Endocr. Rev. 36, 526-563 (2015).

37. Alwashih, M. A. et al. Plasma metabolomic profile varies with glucocorticoid dose in patients with congenital adrenal hyperplasia. Sci. Rep. 7, 17092 (2017).

38. Yamamoto, T. et al. Biochemistry of uridine in plasma. Clin. Chim. Acta 412, 1712-1724 (2011).

39. Lane, A. N. \& Fan, T. W. Regulation of mammalian nucleotide metabolism and biosynthesis. Nucleic Acids Res. 43, 2466-2485 (2015).

40. Deng, Y. et al. An adipo-biliary-uridine axis that regulates energy homeostasis. Science 355, eaaf5375 (2017).

41. Gasser, P. J. \& Lowry, C. A. Organic cation transporter 3: a cellular mechanism underlying rapid, non-genomic glucocorticoid regulation of monoaminergic neurotransmission, physiology, and behavior. Horm. Behav. 104, 173-182 (2018).

42. Lim, M. D. Dried blood spots for global health diagnostics and surveillance: opportunities and challenges. Am. J. Trop. Med. Hyg. 99, 256-265 (2018). 